\title{
Effect of mild $\alpha$-chymotrypsin treatment of highly viscous semen samples on fertilization rates
}

\author{
Andreas Schallmoser ${ }^{1}$, Fadi Bakjaji ${ }^{1}$, Sebastian Königsberger ${ }^{2}$, Julia John $^{1}$, Cara Färber ${ }^{1}$, Elke Schmidt ${ }^{3}$, \\ Hannelore Breitenbach-Koller ${ }^{4}$, Jean-Pierre Allam ${ }^{5}$, Jasper Verguts ${ }^{6}$, Nicole Sänger ${ }^{1}$ \\ ${ }^{1}$ Department of Gynecological Endocrinology and Reproductive Medicine, University Hospital of Bonn, Bonn, Germany; ${ }^{2}$ Eurofins Bio Pharma \\ Munich, Munich, Germany; ${ }^{3}$ Fertility Clinic Tübingen, Tübingen, Germany; ${ }^{4}$ Department of Cell Biology, University of Salzburg, Salzburg, Austria; \\ ${ }^{5}$ Jessa Hospital, Hasselt, Belgium; ${ }^{6}$ Department of Dermatology and Allergy, University Hospital of Bonn, Bonn, Germany \\ Contributions: (I) Conception and design: All authors; (II) Administrative support: All authors; (III) Provision of study materials or patients: All \\ authors; (IV) Collection and assembly of data: All authors; (V) Data analysis and interpretation: All authors; (VI) Manuscript writing: All authors; (VII) \\ Final approval of manuscript: All authors. \\ Correspondence to: Andreas Schallmoser; Nicole Sänger. Universitätsklinikum Bonn, Venusberg Campus 1, 53127 Deutschland, Germany. \\ Email: andreas.schallmoser@ukbonn.de; nicole.saenger@ukbonn.de.
}

\begin{abstract}
Background: Highly viscous semen reduces sperm motility significantly and can contribute to infertility. When processing semen samples, few techniques exist to induce liquefaction in case of seminal hyperviscosity such as different washing steps and mechanical treatment. The use of $\alpha$-chymotrypsin seems controversial due to possible negative effects on fertilisation rates after in vitro fertilization (IVF). The main objective of this study was to examine the influence of mild $\alpha$-chymotrypsin treatment of semen samples on the fertilisation rate after artificial reproductive treatment (ART).

Methods: The fertilization rate of 52 ART cycles was examined following IVF using a low dose of $\alpha$-chymotrypsin to induce liquefaction of highly viscous semen and was compared to a control group of 88 ART cycles.

Results: There was no significant difference in the fertilization rates of $\alpha$-chymotrypsin treated semen samples compared to the control group; pregnancy rates were unaffected.

Conclusions: The use of mild $\alpha$-chymotrypsin treatment of semen samples in case of hyperviscosity does not appear to impact negatively on the fertilization rates after ART and could be regarded as an additional method to induce liquefaction of highly viscous semen samples in IVF.
\end{abstract}

Keywords: Semen; chymotrypsin; viscosity

Submitted Jun 18, 2020. Accepted for publication Oct 28, 2020.

doi: $10.21037 /$ tau-20-1013

View this article at: http://dx.doi.org/10.21037/tau-20-1013

\section{Introduction}

Human semen coagulates shortly after ejaculation. The resulting gelatinous mass is formed by proteins deriving from the seminal vesicles: semenogelin I and semenogelin II, and to a lesser extent by fibronectin (1). Liquefaction occurs 5-20 minutes post ejaculation and is caused by prostate-specific antigen (PSA), a serine protease deriving from the prostate $(2,3)$. The complete sample usually liquefies within 15 minutes at room temperature (4). PSA is a main component of human semen and is a chymotrypsinlike protease $(5,6)$.

High viscosity of semen may contribute to infertility and is observed among $12-29 \%$ of male infertility patients $(7,8)$.

^ ORCID: 0000-0003-1128-2376. 
In a publication by Esfandiari (9) it was demonstrated that seminal hyperviscosity is associated with poor outcome of in vitro fertilization (IVF).

In andrology services and in fertility clinics few strategies exist to induce liquefaction in case of incomplete or lacking semen liquefaction such as different washing steps with sperm preparation media and mechanical processing using injection needles. Chymotrypsins are endoproteases and the use of this mucolytic agents in case of hyperviscous semen samples is described by different authors (10-14).

The use of digestion enzymes like $\alpha$-chymotrypsin seems controversial due to a possible negative effect on the fertilisation rate in IVF. It was demonstrated however that treatment with $\alpha$-chymotrypsin does not impair sperm concentration, motility and velocity and the concentration of gamma-glutamyltranspeptidase, alpha-glucosidase and fructose in seminal plasma samples were unaffected (15). Different authors showed that treatment of viscous semen samples with $\alpha$-chymotrypsin did not impact negatively on the pregnancy rates or led to higher miscarriage rates when performing intrauterine insemination (16-18). With IVF , no aberrations concerning fertilization and pregnancy rate could be observed (11).

There is however growing evidence that treatment of semen samples with $\alpha$-chymotrypsin may alter the expression pattern of sperm functional proteins and potentially affects artificial reproductive treatment (ART) outcome. As sperm surface protein HspA2 is necessary for acrosome reaction and sperm-oocyte interaction $(14,19,20)$, and BAG6, also known as Bat3/Scyth (21) could be identified as a critical regulator of Hsp70-2/HspA2 in spermatogenesis, these proteins were non-detectable in $\alpha$-chymotrypsin-treated semen samples (13).

Based on these suggestions, it is assumed that the use of $\alpha$-chymotrypsin might adversely affect the fertilization potential of human sperm cells due to its high catalytic potential. We hypothesized that the fertilization rate would not be altered after IVF using a low dose of $\alpha$-chymotrypsin with short incubation time. We present the following article in accordance with the STROBE reporting checklist (available at http://dx.doi.org/10.21037/tau-20-1013).

\section{Methods}

All patients were recruited at the Department of Gynecological Endocrinology and Reproductive Medicine, University Hospital of Bonn, Germany from July first 2018 till June first 2019. Anonymized data on age, number of previous pregnancies and deliveries were retrospectively collected. All procedures performed in this study were in accordance with the Declaration of Helsinki (as revised in 2013) and approved by the Ethics Committee of the University Hospital of Bonn, Germany (IRB00001791), approval code 316/19. Because of the retrospective nature of the research and the use of routine methods the requirement for informed consent was waived.

\section{Assessment of semen donations}

Semen quality from male individuals obtaining IVF treatment was assessed via spermiogram analysis according to the WHO laboratory manual (4) for the examination of human semen for spermiogram analysis of the male partner prior to IVF. Normal morphology of $4 \%$, a sperm volume of $1.5 \mathrm{~mL}$, a sperm concentration of $15 \mathrm{Mio} / \mathrm{mL}$ and progressive motility of $32 \%$, resulting in a total number of progressive sperm cells of at least 7.2 Mio were considered as adequate for IVF. Samples falling below these cut-off values were graded as mild male infertility and were still considered for IVF when compensation was reached either through sperm volume, sperm concentration or progressive motility and approved by an experienced embryologist. Semen analysis and assessment of semen samples used for IVF were performed 30 minutes after liquefaction, according to $\mathrm{WHO}$ recommendation (4). A basic analysis was used renouncing staining techniques to determine morphology, vitality and peroxidase test. Sperm volume was measured by decanting the semen into a graduated cylinder, sperm concentration and progressive motility were determined using a Makler Chamber. Total sperm count was calculated by multiplying the sperm concentration with the semen volume of the sample, total progressive sperm count was determined by multiplying the total sperm count with the percent motility.

\section{Routine artificial induction of liquefaction}

Semen viscosity was estimated before and after $\alpha$-chymotrypsin treatment by aspirating the sample into a $3.5-\mathrm{mL}$ wide bore sterile pipette (Sarstedt) allowing the semen to drop with gravity. Ejaculates with abnormal high viscosity formed a thread with a length more than $2 \mathrm{~cm}$ (World Health Organization 2010). Semen samples were also graded as abnormally high viscous in case of non-liquefaction after 30 minutes. Two mg of $\alpha$-chymotrypsin (Merck ${ }^{\circledR}$, Darmstadt, Germany) was added to the ejaculate, mixed and incubated for 5 minutes prior to sperm analysis and sample processing for IVF. No mechanical liquefaction was performed. All samples 
improved significantly and gained liquefaction.

\section{Processing of semen donations for IVF}

Semen samples were processed by separation from seminal plasma using a density gradient system consisting of $1 \mathrm{~mL}$ of a $90 \%$ lower layer $\left(\right.$ Gynemed $^{\circledR}$, Lensahn, Germany), $1 \mathrm{~mL}$ of a $45 \%$ upper layer $\left(\right.$ Gynemed $^{\circledR}$, Lehnsahn, Germany) and $1 \mathrm{~mL}$ of liquefied semen (22). Centrifugation was performed at $300 \mathrm{~g}$ for 15 minutes. The sperm pellet was transferred to another centrifugation tube and washed with $1 \mathrm{~mL}$ sperm rinse media (Vitrolife ${ }^{\circledR}$, Gothenburg, Sweden). Centrifugation was performed for 10 minutes at $300 \mathrm{~g}$, the supernatant was discarded and $1 \mathrm{~mL}$ G-IVF+ media (Vitrolife ${ }^{\circledR}$, Gothenburg, Sweden) was added to the pellet and gently mixed. After centrifugation at $300 \mathrm{~g}$ for 10 minutes the supernatant was extracted, leaving the sperm pellet in the tube with $100 \mu \mathrm{L}$ of G-IVF+ media. The sample was placed in a $6 \% \mathrm{CO}_{2}$ incubator for 15 to 30 minutes allowing the swim-up technique, giving the motile sperm cells the possibility to swim up to the surface from the pellet. The surface fraction was extracted gently without disturbing the pellet and analyzed in a Makler chamber.

\section{Ovarian stimulation protocol}

Stimulation in both protocols was performed by subcutaneous injection of recombinant follicle-stimulating hormone (FSH) (Gonal-F 200 IU, Merck-Serono, Darmstadt, Germany, or Puregon ${ }^{\circledR} 200$ IU, MSD Sharp \& Dohme GmbH, Haar, Germany) or menotropin (Menogon ${ }^{\circledR}$ 225 IU, Ferring, Kiel, Germany). Downregulation was obtained following either a long protocol by using of intranasal buserelin acetate $(0.156 \mathrm{mg} 4$ times daily, Ferring, Kiel, Germany) or subcutaneous triptorelin acetate (100 $\mu \mathrm{g}$ daily, Ferring, Kiel, Germany). In the short protocol ganirelix (Orgalutran ${ }^{\circledR} 0.25 \mathrm{mg}$ daily, MSD Sharp \& Dohme GmbH, Haar, Germany) was used. The dose of FSH was adjusted according to individual response. Human chorionic gonadotropin (hCG) (Ovitrelle ${ }^{\circledR}$ 6,500 IU, Merck-Serono, Darmstadt, Germany) was administered when at least three follicles of minimum $17 \mathrm{~mm}$ were visible, and about $36 \mathrm{~h}$ later pick-up was performed.

\section{IVF and embryo culture}

Cumulus oocyte complexes (COC) were retrieved by follicular aspiration and inseminated by adding at least
100,000 motile sperm cells to up to 3 COCs. After denudation, pronuclear (PN) scoring was performed 16 to $18 \mathrm{~h}$ after the IVF procedure. Cells were cultured in single droplets with $20 \mu \mathrm{L}$ of cell culture media in humidified IVF incubators at $37{ }^{\circ} \mathrm{C}$, with $6 \% \mathrm{CO}_{2}, 5 \% \mathrm{O}_{2}$ and $89 \% \mathrm{~N}_{2}$. In case of ovarian hyperstimulation (OHSS) or other medical indications all fertilized cells of the treatment cycle were frozen directly after PN scoring (freeze all).

\section{Embryo transfer and pregnancy}

Embryo transfer was performed at day 3 or 5 (fresh embryo transfer procedure). Serum hCG levels were assessed after 14 days. Clinical pregnancy rate was defined by the presence of a fetal heartbeat at 6-7 weeks of pregnancy. Biochemical pregnancy was specified as the absence of a clinical pregnancy. hCG value might be negative or, e.g., start of menstruation ahead of planned ultrasound examination.

\section{Statistical analysis}

Data was analyzed using SPSS version $25\left(\right.$ IBM $^{\circledR}$ Armonk, New York, USA). A Mann-Whitney U test was used to compare continuous parameters between groups. The relationship between categorial variables was analyzed with the Chi-square test. A $t$-test was used to determine differences between the means of both groups. Significance level was set at $5 \%$.

\section{Results}

The study group consisted of a cohort of 52 couples meeting the inclusion criteria of highly viscous semen and were compared to a cohort control group of 88 couples.

The fertilization rate between the two groups was not significant $(\mathrm{P}=0.146$, Mann-Whitney $\mathrm{U}$ test), with a rate of 57.95 in the study group compared to 57.53 in the control group (Table 1).

Our analysis showed no significant differences in male and female age, male abstinence period, semen volume, sperm concentration, total sperm count, and total progressive sperm count between the two groups (Table 2). We observed a significant difference $(\mathrm{P}=0.025)$ in the progressive motility and a borderline significance in the total progressive sperm count $(\mathrm{P}=0.052)$ between $\alpha$-chymotrypsin treated samples and the non- $\alpha$-chymotrypsin treated samples.

Analyzing the clinical and biochemical pregnancy parameters of the study group $(n=41)$ and the control group 
Table 1 Fertilization rate in relation to mild $\alpha$-chymotrypsin treatment of semen samples

\begin{tabular}{lccc}
\hline Parameter & Study group $(n=52)$ & Control group $(n=88)$ & $P$ value* \\
\hline Fertilization rate (per oocyte inseminated) & 57.95 & 57.53 & 0.146 \\
\hline
\end{tabular}

*, Mann-Whitney U test, $\mathrm{n}=140$ samples.

Table 2 Age and basic semen parameters in relation to mild $\alpha$-chymotrypsin treatment of semen samples

\begin{tabular}{lccc}
\hline Parameter & Study group $(\mathrm{n}=52)$ & Control group $(\mathrm{n}=88)$ & $P$ value* \\
\hline Semen volume $(\mathrm{mL})$ & $3.0(2.5-3.5)$ & $3.2(2.7-3.8)$ & 0.190 \\
Sperm concentration $(\mathrm{Mio} / \mathrm{mL})$ & $62.0(49.0-86.0)$ & $77.0(70.0-84.0)$ & 0.303 \\
Progressive motility (\%) & $39.5(34.0-43.0)$ & $44.50(42.5-48.5)$ & 0.025 \\
Total sperm count (Mio) & $179.0(144.0-250.1)$ & $223.0(179.0-257.2)$ & 0.163 \\
Total progressive sperm count (Mio) & $64.8(48.5-109.2)$ & $88.15(72.8-119.2)$ & 0.052 \\
Abstinence period (days) & $5.9(4.6-7.1)$ & $5.2(4.8-5.6)$ & 0.613 \\
Female age (years) & $36.7(35.4-38.0)$ & $37.0(36.0-37.9)$ & 0.574 \\
Male age (years) & $39.5(38.0-41.0)$ & $39.4(38.0-40.8)$ & 0.697 \\
\hline
\end{tabular}

*, Mann-Whitney U test, $\mathrm{n}=140$ samples.

Table 3 Pregnancy rate in relation to mild $\alpha$-chymotrypsin treatment of semen samples (in \% per fresh embryo transfer procedure)

\begin{tabular}{lccc}
\hline Parameter & $\begin{array}{c}\text { Study group } \\
\text { (41 embryo transfer procedures) }\end{array}$ & $\begin{array}{c}\text { Control group } \\
\text { (66 embryo transfer procedures) }\end{array}$ & $\begin{array}{l}\mathrm{P} \text { value* } \\
\text { Clinical pregnancy rate (\%) }\end{array}$ \\
Biochemical pregnancy rate (\%) & 29.26 & 27.27 & 0.825 \\
\hline
\end{tabular}

*, $t$-test, $\mathrm{n}=107$ samples.

Table 4 Female infertility parameters: previous pregnancies and childbirth

\begin{tabular}{lccc}
\hline Parameter & Study group $(n=52)$ & Control group $(n=88)$ & $P$ value* \\
\hline Previous pregnancies (\%) & 40.1 & 52.2 & 0.221 \\
Childbirth (\%) & 26.9 & 27.2 & 0.964 \\
\hline
\end{tabular}

*, Chi-square Test, $\mathrm{n}=140$ samples.

$(\mathrm{n}=66)$ per fresh embryo transfer procedures $(\mathrm{n}=107)$ we found no significant differences (Table 3). Freeze all cycles were excluded from the study.

No significant differences concerning previous pregnancies and childbirth were detected (Table 4).

\section{Discussion}

Our analysis showed no significant difference in the fertilization rate of $\alpha$-chymotrypsin treated sperm cells in comparison to an untreated control group.

After IVF of 245 COCs with $\alpha$-chymotrypsin treated sperm cells we observed a fertilization rate of $57.95 \%$ compared to a fertilization rate of $57.53 \%$ in the non$\alpha$-chymotrypsin treated group after IVF of 584 COCs. Fertilization rate of each group was calculated per inseminated oocyte. We observed a significant difference in the progressive motility rate and differences in sperm concentrations between $\alpha$-chymotrypsin treated viscous semen samples and non- $\alpha$ chymotrypsin treated samples as reported by Esfandiari et al. (9) 
who analyzed the fertilization rate of IVF inseminations of viscous semen samples and an untreated control group without the use of digestion enzymes.

In our study, we detected higher values for progressive motility and higher sperm concentrations in both groups that may explain in addition to mild $\alpha$-chymotrypsin treatment of the study group our relatively unaffected fertilization rate. The significant difference in the progressive motility between the two groups could be explained by the higher abstinence period in the $\alpha$-chymotrypsin treated group but this finding was not significant.

We follow the argumentation of Chen et al. (15) who excluded a negative effect of $\alpha$-chymotrypsin treatment on the progressive motility by splitting up semen samples analyzing the progressive motility with and without enzyme treatment. Analyzing the fertilization rate of 14,843 cycles of IVF , the German IVF register (DIR) (23) reported a fertilization rate of $55.1 \%$. These data do not deviate significantly from our fertilization results that were moderately higher in the study group $(57.95 \%, 52$ cycles) and the control group $(57.53 \%, 88$ cycles).

Our results confirm findings of Honea et al. (11), observing no negative effect on the fertilization rates performing IVF with $\alpha$-chymotrypsin treated semens samples on 65 eggs reporting a fertilization rate of $63 \%$.

We found no significant difference in the clinical and biochemical pregnancy rates between the two treatment groups. Mild $\alpha$-chymotrypsin treatment of highly viscous semen samples was not found to have a negative effect on the pregnancy rate, this finding is in concordance with published data $(11,16-18)$ but show partially limited comparability with our results as the majority of the above mentioned studies were treating semen samples with $\alpha$-chymotrypsin prior to intrauterine inseminations where processed sperm was placed directly in the uterus.

In summary, the non-decreased fertilization rate after IVF of $\alpha$-chymotrypsin treated sperm cells does not support suggestions of a potential negative impact on the fertilization rate when low doses of $\alpha$-chymotrypsin are used in combination with short incubation time. The usage of high doses of $\alpha$-chymotrypsin combined with prolonged incubation time may contribute to the effects observed by Panner Selvam et al. (13) To discriminate between these factors, a trial with a higher sample size would be required.

\section{Limitations}

Different weaknesses in the design of this study deserve a comment. Data collection was conducted retrospectively and was not randomized. The study is a pilot study as the majority of the studies using $\alpha$-chymotrypsin was conducted up to three decades ago, comparison of data must be interpreted in the light of the fact that since then, IVF has seen a rapid evolution of technology and culture techniques. To analyse the effects of $\alpha$-chymotrypsin on a specific semen sample it is necessary to split each sample into an $\alpha$-chymotrypsin treated and a non- $\alpha$-chymotrypsin treated group as reported by Chen et al. (15), but this would cause interferences with the internal workflow and was therefore not feasible. In our study, all collected COCs were inseminated and the fertilization rate was calculated per retrieved COC as also performed by Bhattacharya et al. (24). This includes potentially immature oocytes and lowers the fertilization rate compared to a study (25) excluding immature oocytes on day 1 post insemination. Due to German law only limited numbers of $2 \mathrm{PN}$ stages are allowed for embryo culture, and surplus PN stages are frozen. Vitrification of blastocysts is only legally allowed in medical emergencies such as OHSS. This lowers the pregnancy rate significantly in comparison to, e.g., European IVF centres where all PN stages of the same treatment cycle are cultivated and the remaining blastocysts are transferred or frozen on day 5. Our pregnancy rate was calculated per fresh embryo transfer procedures, as we are currently converting our cryopreservation system from slow freezing to vitrification.

\section{Acknowledgments}

Funding: None.

\section{Footnote}

Reporting Checklist: The authors have completed the STROBE reporting checklist. Available at http://dx.doi. org/10.21037/tau-20-1013

Data Sharing Statement: Available at http://dx.doi. org/10.21037/tau-20-1013

Conflicts of Interest: All authors have completed the ICMJE uniform disclosure form (available at http://dx.doi. org/10.21037/tau-20-1013). The authors have no conflicts of interest to declare.

Ethical Statement: The authors are accountable for all 
aspects of the work in ensuring that questions related to the accuracy or integrity of any part of the work are appropriately investigated and resolved. All procedures performed in this study were in accordance with the Declaration of Helsinki (as revised in 2013) and approved by the Ethics Committee of the University Hospital of Bonn, Germany (IRB00001791), approval code 316/19. Because of the retrospective nature of the research and the use of routine methods the requirement for informed consent was waived.

Open Access Statement: This is an Open Access article distributed in accordance with the Creative Commons Attribution-NonCommercial-NoDerivs 4.0 International License (CC BY-NC-ND 4.0), which permits the noncommercial replication and distribution of the article with the strict proviso that no changes or edits are made and the original work is properly cited (including links to both the formal publication through the relevant DOI and the license). See: https://creativecommons.org/licenses/by-nc-nd/4.0/.

\section{References}

1. de Lamirande E. Semenogelin, the main protein of the human semen coagulum, regulates sperm function. Semin Thromb Hemost 2007;33:60-8.

2. Gupta N, Sudhakar DVS, Gangwar PK, et al. Mutations in the prostate specific antigen (PSA/KLK3) correlate with male infertility. Sci Rep 2017;7:11225.

3. Henkel RR, Schill WB. Sperm preparation for ART. Reprod Biol Endocrinol 2003;1:108.

4. World Health Organization. WHO laboratory manual for the examination and processing of human semen. 5th edition. Genève, Suisse: WHO press, 2010.

5. Veveris-Lowe TL, Kruger S, Walsh T, et al.Seminal fluid characterization for male fertility and prostate cancer: kallikrein-related serine proteases and whole proteome approaches. Semin Thromb Hemost 2007;33:87-99.

6. Rittenhouse HG, Finlay JA, Mikolajczyk SD, et al. Human Kallikrein 2 (hK2) and prostate-specific antigen (PSA): two closely related, but distinct, kallikreins in the prostate. Crit Rev Clin Lab Sci 1998;35:275-368.

7. Andrade-Rocha FT. Physical analysis of ejaculate to evaluate the secretory activity of the seminal vesicles and prostate. Clin Chem Lab Med 2005;43:1203-10.

8. Du Plessis SS, Gokul S, Agarwal A. Semen hyperviscosity: causes, consequences, and cures. Front Biosci (Elite Ed) 2013;5:224-31.
9. Esfandiari N, Burjaq H, Gotlieb L, et al. Seminal hyperviscosity is associated with poor outcome of in vitro fertilization and embryo transfer: a prospective study. Fertil Steril 2008;90:1739-43.

10. Di Cera E. Serine proteases. IUBMB Life 2009;61:510-5.

11. Honea KL, Houserman VL, Merryman DC, et al. Effect of limited proteolysis with alpha-chymotrypsin on semen with an abnormal sperm penetration assay and possible application for in vitro fertilization or intrauterine insemination. J Assist Reprod Genet 1993;10:255-60.

12. Zavos P, Correa J, Zannakoupis-Zavos P. Effect of treatment of seminal viscosity difficulties with $\alpha$-chymotrypsin on the recovery of spermatozoa for assisted reproductive technologies: comparison between the SpermPrep'" filtration and Percof gradient centrifugation methods. Middle East Fertil Soc J 1997;2:223-9.

13. Panner Selvam MK, Agarwal A, Sharma R, et al. Treatment of semen samples with $\alpha$-chymotrypsin alters the expression pattern of sperm functional proteins-a pilot study. Andrology 2018;6:345-50.

14. Katsoff D, Check J, Bollendorf A, et al. Chymotrypsin Galactose Treatment of Sperm with Antisperm Antibodies results in improved pregnancy rates following in Vitro Fertilization. Am J Reprod Immunol 1995;33:149-54.

15. Chen F, Lu J, Xu H, et al. Chymotrypsin effects on the determination of sperm parameters and seminal biochemistry markers. Clin Chem Lab Med 2006;44:1335-9.

16. Check JH, Hourani W, Check M, et al. Effect of treating antibody-coated sperm with chymotrypsin on pregnancy rates following IUI as compared to outcome of IVF/ICSI. Arch Androl. 2004;50:93-5.

17. Bollendorf A, Check J, Katsoff D, et al. The use of chymotrypsin/galactose to treat spermatozoa bound with anti-sperm antibodies prior to intra-uterine insemination. Hum Reprod 1994;9:484-8.

18. Freischem CW, Bordt J, Hanker JP, et al. Pregnancy after treatment of ejaculate with alpha-chymotripsine because of failure to liquefy. Geburtshilfe Frauenheilkd 1983;43:490-1.

19. Redgrove KA, Nixon B, Baker MA, et al. The molecular chaperone HSPA2 plays a key role in regulating the expression of sperm surface receptors that mediate spermegg recognition. PLoS One 2012;7:e50851.

20. Redgrove KA, Anderson AL, McLaughlin EA, et al. Investigation of the mechanisms by which the molecular chaperone HSPA2 regulates the expression of sperm surface receptors involved in human sperm-oocyte 
recognition. Mol Hum Reprod 2013;19:120-35.

21. Lee JG, Ye Y. Bag6/Bat3/Scythe: A novel chaperone activity with diverse regulatory functions in protein biogenesis and degradation. Bioessays 2013;35:377-85.

22. Schallmoser A, Raab M, Karn T, et al. Quantitative analysis of the sHLA-G protein in seminal plasma. Am J Reprod Immunol 2019;82:e13152.

23. Blumenauer V, Czeromin U, Fehr D, et al. D.I.RAnnual 2018. Journal of Reproductive Medicine and Endocrinology 2019;16:272-311.

Cite this article as: Schallmoser A, Bakjaji F, Königsberger S, John J, Färber C, Schmidt E, Breitenbach-Koller H, Allam JP, Verguts J, Sänger N. Effect of mild $\alpha$-chymotrypsin treatment of highly viscous semen samples on fertilization rates. Transl Androl Urol 2021;10(1):448-454. doi: 10.21037/tau-20-1013
24. Bhattacharya S, Hamilton M, Shaaban M. et al. Conventional in-vitro fertilisation versus intracytoplasmic sperm injection for the treatment of non-male-factor infertility: a randomised controlled trial. Lancet 2001;357:2075-9.

25. Foong SC, Fleetham JA, O'Keane JA, et al. A prospective randomized trial of conventional in vitro fertilization versus intracytoplasmic sperm injection in unexplained infertility. J Assist Reprod Genet 2006;23:137-40. 